\title{
\begin{tabular}{l|l} 
Mitraries & DSpace@MIT
\end{tabular}
}

\author{
MIT Open Access Articles
}

Ultrabroadband 2D electronic spectroscopy as a tool for direct visualization of pathways of energy flow

The MIT Faculty has made this article openly available. Please share how this access benefits you. Your story matters.

Citation: Schlau-Cohen, Gabriela, and Son, Minjung Son “Ultrabroadband 2D Electronic Spectroscopy as a Tool for Direct Visualization of Pathways of Energy Flow." Edited by Hugo A. Bronstein and Felix Deschler. Physical Chemistry of Semiconductor Materials and Interfaces XVI (August 2017) (c) 2017 SPIE

As Published: http://dx.doi.org/10.1117/12.2273417

Publisher: SPIE

Persistent URL: http://hdl.handle.net/1721.1/113596

Version: Final published version: final published article, as it appeared in a journal, conference proceedings, or other formally published context

Terms of Use: Article is made available in accordance with the publisher's policy and may be subject to US copyright law. Please refer to the publisher's site for terms of use. 


\section{Ultrabroadband 2D electronic spectroscopy as a tool for direct visualization of pathways of energy flow}

Minjung Son, Gabriela S. Schlau-Cohen

Minjung Son, Gabriela S. Schlau-Cohen, "Ultrabroadband 2D electronic spectroscopy as a tool for direct visualization of pathways of energy flow," Proc. SPIE 10348, Physical Chemistry of Semiconductor Materials and Interfaces XVI, 1034817 (24 August 2017); doi: 10.1117/12.2273417

Event: SPIE Nanoscience + Engineering, 2017, San Diego, California, United States 


\title{
Ultrabroadband 2D electronic spectroscopy as a tool for direct visualization of pathways of energy flow
}

\author{
Minjung Son and Gabriela S. Schlau-Cohen \\ Department of Chemistry, Massachusetts Institute of Technology, 77 Massachusetts Avenue, \\ Cambridge, MA 02139, United States
}

\begin{abstract}
Two-dimensional electronic spectroscopy (2DES) has emerged as an incisive tool for mapping out energy relaxation pathways in complex molecular systems by determining correlation maps between the excitation and emission frequencies. Its enhanced spectral as well as temporal resolution offer new insights into coupling and energy transfer between closely-spaced energy states, which are often hidden in a one-dimensional transient spectrum. However, a major drawback of the current 2DES technique is that the spectral window of detection is directly limited to the laser bandwidth used, which leads to an incomplete visualization of the full energy landscape of the system. As a solution to this limitation, we present an ultrabroadband 2DES apparatus utilizing a 8 -fs, 185-nm bandwidth supercontinuum that covers the entire visible region. We demonstrate the utility of our setup by measuring the 2D spectra of laser dyes absorbing at different regions of the laser spectrum, and the major light-harvesting complex of spinach.
\end{abstract}

Keywords: Multidimensional spectroscopy, ultrafast spectroscopy, photosynthesic light harvesting

\section{INTRODUCTION}

Photoexcitation of light-absorbing molecules induces a wide variety of photophysical and photochemical dynamics with time scales ranging from femtoseconds (energy transfer, electron transfer) to nanoseconds (fluorescence emission, rotational motion) or even microseconds (phosphorescence). ${ }^{1}$ Among these time scales, we are often particularly interested in elucidating the early-time energy relaxation pathways of the system immediately following photoabsorption, which often involves energy transfer and/or internal conversion processes as fast as a few tens of femtoseconds. While the development of ultrafast spectroscopic techniques, such as transient absorption $^{2}$ and time-resolved fluorescence, ${ }^{3}$ greatly aided in revealing the kinetics of such ultrafast dynamics, correlating the observed rate constants with temporal evolution of the spectral signatures remains non-trivial due to the inability of these techniques to simultaneously measure with high temporal and energy (frequency) resolution. Specifically, this becomes a major challenge in the studies of complex condensed-phase systems, e.g. photosynthetic light harvesting complexes, because their congested and highly delocalized energy manifolds lead to a significant overlap in the spectral signatures, obscuring the pathways of energy transfer. ${ }^{4-6}$

Coherent two-dimensional (2D) optical spectroscopy is an advanced spectroscopic technique that is capable of resolving both time and energy by spreading out the third-order signal along one frequency axis (emission frequency, $\omega_{t}$ ) onto a second frequency axis (excitation frequency, $\omega_{\tau}$ ) as a function of a delay time between the excitation and emission events. 2D spectroscopy constructs a correlation map of $\omega_{\tau}$ and $\omega_{t}$, which enables direct observation of the couplings between states and the energy relaxation pathways of the system. ${ }^{4,7}$ This combination of enhanced spectral and temporal resolutions makes 2D optical spectroscopy a powerful tool for interrogating the energy landscapes and dynamics of condensed-phase systems. In the visible regime, twodimensional electronic spectroscopy (2DES) has been extensively utilized to map out energy relaxation pathways in a vast array of systems including photosynthetic light harvesting complexes, ${ }^{5,8-10}$ molecular aggregates, ${ }^{11-13}$ and inorganic and organic semiconductors. ${ }^{14-18}$

Send correspondence to G.S.S.-C.

G.S.S.-C.: E-mail: gssc@mit.edu, Telephone: +1-617-253-1478

Physical Chemistry of Semiconductor Materials and Interfaces XVI, edited by Hugo A. Bronstein, Felix Deschler, Artem A. Bakulin, Proc. of SPIE Vol. 10348, 1034817 · @ 2017

SPIE · CCC code: $0277-786 \mathrm{X} / 17 / \$ 18 \cdot$ doi: $10.1117 / 12.2273417$ 
Nonetheless, one of the remaining problems in the vast majority of $2 \mathrm{D}$ electronic spectra reported to date is that the spectral window of detection is greatly limited due to the limited bandwidth of the laser spectrum, which is typically generated by non-collinear optical parametric amplifiers (NOPAs). ${ }^{19}$ While NOPAs offer tunable wavelength range across the visible region, their bandwidth is limited to sub-100 $\mathrm{nm}$, constraining the detection window to only several tens of nanometers. ${ }^{20}$ Consequently, the utility of 2DES has been limited to uncovering energy flow over a narrow range of transitions, which provides an incomplete picture of the energy landscape. To overcome this limitation, ultrabroadband, few-cycle pulses generated by gas filamentation ${ }^{21,22}$ are being implemented in the most recent 2DES apparatuses, ${ }^{23-26}$ which enables simultaneous probing of a broad range of electronic and vibronic transitions across the entire visible region.

In this article, we present our recently developed ultrabroadband 2DES setup with 8-fs, 185-nm bandwidth pulses spanning the entire visible region $(450-800 \mathrm{~nm})$ and its application to energy transfer processes in the major light harvesting complex (light-harvesting complex II, LHCII) of higher plants. While previous studies on energy transfer in LHCII were localized on the energy migration in and out of the two lowest-energy $\left(Q_{y}\right)$ bands of chlorophylls, ${ }^{27-30}$ the enhanced spectral coverage of our apparatus allows us to follow additional energy relaxation pathways from higher energy states into these states. We first give a brief introduction of the principles of 2DES, followed by a detailed description of our 2DES apparatus. We demonstrate the functionality of our ultrabroadband 2DES setup by presenting the 2D spectra of (1) two laser dyes, rhodamine 6G (R6G) and Nile Blue A perchlorate (NB), and (2) LHCII of spinach.

\section{MATERIALS AND METHODS}

\subsection{Two-Dimensional Electronic Spectroscopy (2DES)}

2DES is a third-order nonlinear spectroscopic technique, in which three laser pulses interact with the system of interest to induce emission of a signal. The pulse sequence of a 2DES experiment is illustrated in Figure 1A. The first pulse (1) excites the sample and creates a coherence between the ground state and the first excited state of the system, which evolves during the coherence time $(\tau)$. When the second pulse $(2)$ enters the sample, the system freely evolves during the waiting time $(T)$ until the third pulse (3) arrives, which generates a second coherence and records the current state of the system, as the probe pulse does in one-dimensional pump-probe spectroscopy. ${ }^{6,20}$ The signal is emitted after a time delay $t$, which is heterodyne detected by interfering with the last pulse, commonly referred to as local oscillator (LO). The LO does not directly interact with the system, but serves to amplify the emitted signal and enables the extraction of both the amplitude and phase of the complex signal field. ${ }^{20}$ To construct the frequency-frequency correlation map $\left(\omega_{\tau}, \omega_{t}\right)$, the excitation frequency axis $\left(\omega_{\tau}\right)$ is retrieved by Fourier transformation of the collected time-domain data $(\tau)$, whereas the emission frequency axis $\left(\omega_{t}\right)$ is directly obtained by recording the spectral interference between the signal and the LO.

\subsection{Experimental Setup}

The optical layout of our ultrabroadband 2DES setup is shown in Figure 1B. The ultrabroadband light source is generated by focusing an attenuated output of a $5-\mathrm{kHz}$ Ti:sapphire regenerative amplifier (Coherent Libra, centered at $800 \mathrm{~nm}, 32-\mathrm{nm}$ bandwidth, $<40$ fs duration) into a 1-m chamber filled with pressurized argon gas $(20 \mathrm{psi}),{ }^{23,24}$ which induces self-phase modulation. After initial spectral broadening by argon filamentation, a combination of a shortpass dichroic mirror (DCM) and colored glass filters (GF) are employed to produce the final ultrabroadband spectrum (Figure 1C), which filter out the intense residual from the fundamental $(750-850$ $\mathrm{nm})$. The final output pulse spans the entire visible region $(450-800 \mathrm{~nm})$ with a full-width at half maximum (FWHM) bandwidth of $185 \mathrm{~nm}$ (centered at $610 \mathrm{~nm}$ ) and a maximum pulse energy of $12 \mu \mathrm{J}$. Two pairs of group velocity delay (GVD)-oscillation-compensated chirped mirrors (CM1 and CM2) are utilized to compress the ultrabroadband pulse to $8 \mathrm{fs}^{31}$ The temporal profile of the pulse is characterized by measuring transient grating frequency-resolved optical gating (TG-FROG) ${ }^{32}$ on a $0.2-\mathrm{mm}$ path-length quartz cuvette filled with methanol as the nonlinear medium (Figure 1D). The compressed ultrabroadband pulse then enters the 2DES setup.

Because of the extremely broad bandwidth of our pulse, the use of diffractive and transmissive optics was minimized throughout the entire 2DES apparatus. ${ }^{20}$ The four beams are obtained by two beamsplitters (BS1 and BS2) in the background-free, BOXCARS geometry. The phase stability of our apparatus is measured to be $\lambda / 146$ over 20 minutes and $\lambda / 75$ over 10 hours. A retroreflector (RR1) mounted on a motorized translational stage 
A

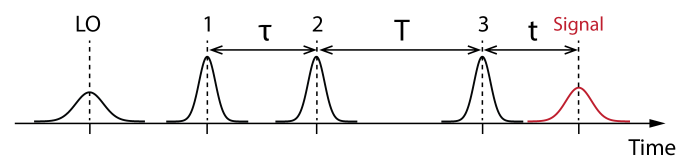

B

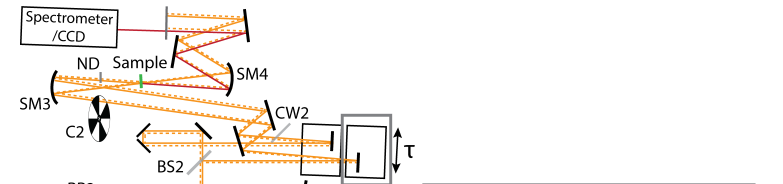

C

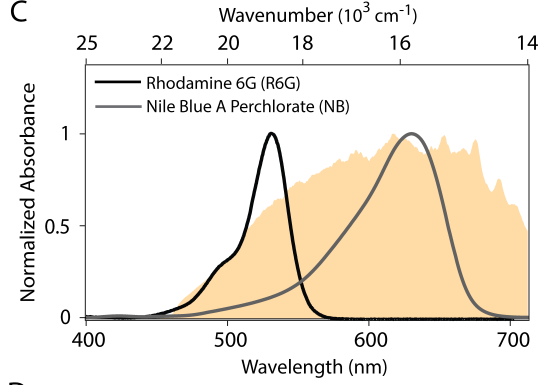

D

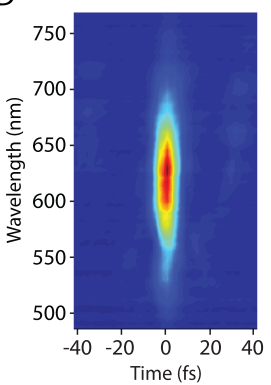

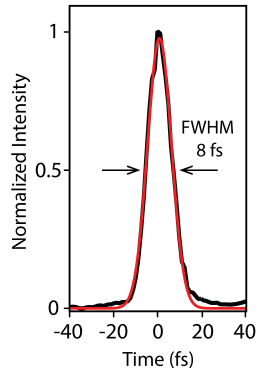

Figure 1. (A) Pulse sequence of a 2DES experiment. Pulses $1-3$ sequentially interacts with the sample and generate the third-order nonlinear signal, which is heterodyne-detected by mixing with the local oscillator (LO). (B) Optical layout of the ultrabroadband 2DES setup. Path of the fundamental pulse is shown in red, the ultrabroadband laser pulses are shown in orange, and the signal is shown in dark red. L1 and L2 - focusing and collimating lenses $(f=1000 \mathrm{~mm})$; DCM - shortpass dichroic mirror (805 nm cutoff); GF - colored glass filters; CM1 and CM2 - chirped mirror pairs; SM1 and SM2 - spherical (concave) mirrors $(f=150 \mathrm{~mm}$ and $75 \mathrm{~mm}$ ); SM3 and SM4 - spherical (concave) mirrors $(f=250 \mathrm{~mm})$; BS1 and BS2 - 50:50 beam splitters; CW1 and CW2 - compensating windows (1-mm UV fused silica); RR1 and RR2 retroreflectors; $\mathrm{C} 1$ and $\mathrm{C} 2$ - optical choppers; ND - neutral density filter (OD 3). Inset shows detailed illustration of the ARID assembly (only one nanopositioner stage is shown for clarity). $\Delta d$ : actual increment of the nanopositioner, $\Delta x$ : effective increment of the nanopositioner retrieved from the relationship $\Delta x=\Delta d \sin \theta$. (C) Linear absorption spectra of rhodamine 6G (R6G, black) and Nile Blue A perchlorate (NB, gray) overlaid with the laser spectrum (orange area). (D) Left: TG-FROG trace of the ultrabroadband pulse measured at the sample position. Right: Retrieved temporal profile (black) and the fit to a Gaussian function (red).

controls the waiting time $(T)$, the time delay between beams 2 and 3 (see Figure 1A for the pulse sequence of a 2DES experiment). For precise control of the coherence time delay $(\tau)$, we adopt the all-reflective interferometric delay (ARID) system developed by Zheng et al (inset of Figure 1B).$^{23}$ Briefly, the system consists of two nanopositioner stages mounted at an angle $(\theta)$ to the plane normal to the beam propagation, each with a mirror mounted on top that reflects beam 1 and 2, respectively. Choosing a small angle $\left(\theta=\sim 0.3^{\circ}\right.$ in our case), the effective increment of the coherence time steps $(\Delta x)$ is reduced by nearly $\left(1 / \sin 0.3^{\circ}\right) \approx 190$ times from the actual increment of the stage $(\Delta d)$. This allows us to vary $\tau$ with a comparable or better precision compared to wedge pair delays, ${ }^{33,34}$ while introducing no further dispersion into the 2DES apparatus. In addition to the two mirrors mounted on the nanopositioners, two mirrors mounted above them reflect beam 3 and the LO, forming a four-quadrant mirror system as reported by Zhang et al. ${ }^{35}$ The four beams are focused into a $100-\mu$ m diameter spot at the sample position using concave mirrors. A neutral density filter is inserted in the path of the LO, which attenuates the LO intensity as well as introduces a temporal delay between the LO and the other three pulses. Due to the phase matching geometry, the emitted third-order nonlinear signal travels in the direction of the LO. The signal and the LO are directed into a home-built spectrometer where a line-scan charge-coupled device (CCD) detects the spectral interference of the two. To achieve better detection sensitivity, we employ a $5-\mathrm{kHz}$ shot-to-shot, dual-chopping scheme for the detection of our 2DES data. Namely, image acquisition of the $\mathrm{CCD}$ is synchronized to the laser repetition rate $(5 \mathrm{kHz})$ so that it collects every laser shot. At the same time, two optical choppers ( $\mathrm{C} 1$ and $\mathrm{C} 2$ ) are also slaved to the laser trigger, which enables real-time subtraction of scatter by sequentially blocking different beams at subharmonics of the laser frequency $(\mathrm{C} 1: 2.5 \mathrm{kHz}, \mathrm{C} 2: 1.25$ 
$\mathrm{kHz}){ }^{36}$ This ensures that the acquired data are free from background originating from laser fluctuation as well as artifacts caused by scatter.

\subsection{Sample Preparation and Experimental Details}

The two dyes, rhodamine 6G and Nile Blue A perchlorate were purchased from Sigma-Aldrich and used without further purification. Both dyes were dissolved in ethanol to an optical density (OD) of 0.25 in a 0.1-mm pathlength quartz cuvette. Trimeric LHCII was isolated from spinach according to the procedure described in Caffari et al., ${ }^{37}$ solubilized in $10 \mathrm{mM}$ HEPES, $20 \mathrm{mM} \mathrm{NaCl}(\mathrm{pH}=7.5)$ containing $0.03 \% \alpha$-dodecylmaltoside $(\alpha$-DM), and stored at $-80^{\circ} \mathrm{C}$ until further use. The samples were thawed and diluted with the buffer solution immediately before the measurement. The OD at the $\mathrm{Chl} a \mathrm{Q}_{\mathrm{y}}$ maximum $(675 \mathrm{~nm})$ was measured to be 0.5 per $0.1 \mathrm{~mm}$.

For 2DES measurements, the pulse energy was attenuated to $15 \mathrm{~nJ} /$ pulse before entering the sample using a neutral density filter. The LO was further attenuated by an OD $=3$ neutral density filter, and temporally delayed from beams $1-3$ by $\sim 500$ fs using a manual translational stage. In all measurements, the coherence time $(\tau)$ was scanned in the range of $-120-120 \mathrm{fs}$ with a step size of $0.4 \mathrm{fs}$ at each fixed waiting time $(T)$. The rephasing $\left(k_{R}=-k_{1}+k_{2}+k_{3}\right)$ and non-rephasing signals $\left(k_{N R}=k_{1}-k_{2}+k_{3}\right)$ were collected by reversing the timing between pulses 1 and 2 by moving the corresponding nanopositioner. For phasing of the 2D spectra, auxiliary pump-probe spectra were collected after each set of 2DES measurement, using beam 2 as the pump and the LO as the probe. The pump-probe spectra were also acquired shot-to-shot by blocking beam 2 every other shot using the 2.5-kHz chopper $(\mathrm{C} 1)$. To obtain the real (absorptive) 2D spectra, the raw 2D data were phased according to the projection slice theorem, ${ }^{7}$ where two phase correction factors were applied to account for linear and quadratic errors in the time delay between beam 3 and the LO. To prevent slow photobleaching of the sample, the LHCII sample was flowed in a $0.1-\mathrm{mm}$ path-length flow cell at a rate of $0.4 \mathrm{~mL} / \mathrm{s}$. All measurements of the LHCII sample were performed at $4^{\circ} \mathrm{C}$.

\section{RESULTS}

\subsection{Ultrabroadband 2D electronic spectroscopy of laser dyes}

The functionality of our ultrabroadband 2DES setup was examined by measuring the $2 \mathrm{D}$ spectra of two laser dyes, rhodamine 6G (R6G) and Nile Blue A perchlorate (NB) as test samples. Because our ultrabroadband pulse can resonantly excite the main absorption bands of both dyes simultaneously $\left(\lambda_{\max }=530 \mathrm{~nm}\right.$ for R6G, $630 \mathrm{~nm}$ for NB, see Figure 1C), we measured the two samples sequentially without changing the pulse condition. Representative absorptive 2D spectra of the dyes are shown in Figure 2A.
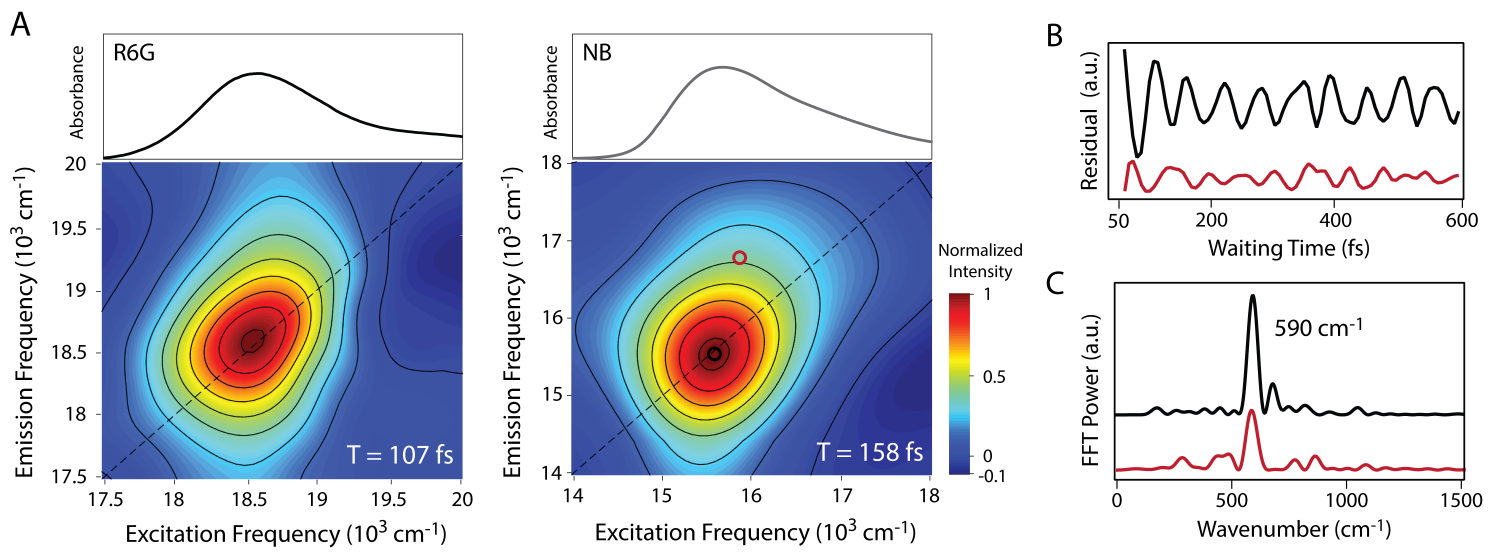

Figure 2. (A) Real (absorptive) 2D spectra of R6G (left) and NB (right) at indicated waiting times $(T)$. Dye absorption spectra are also shown above each 2D spectrum. (B) Plot of oscillations along waiting time observed for NB. Pure wave packet motions were obtained by fitting the raw $2 \mathrm{D}$ signal along $T$ with an exponential function and subtracting the exponential decay component. The two exemplary points monitored are marked as circles in $(\mathrm{A}):\left(\omega_{\tau}, \omega_{t}\right)=(15600$ $\left.\mathrm{cm}^{-1}, 15500 \mathrm{~cm}^{-1}\right)$ (black); (15820 $\left.\mathrm{cm}^{-1}, 16840 \mathrm{~cm}^{-1}\right)$ (red). (C) FFT power spectra of the oscillatory traces in (B). 
Both spectra exhibit structureless, positive peaks along the diagonal, in agreement with previously reported results on these dyes. ${ }^{26,33,38}$ In both cases the peak positions match the absorption maxima of the dyes, which indicates that the 2D spectra are dominated by ground-state bleach (GSB) of the dyes. In addition, from the intensity of $2 \mathrm{D}$ signal plotted along $T$ we were able to observe vibrational wave packet motions of NB (Figure 2B,C), which appear as oscillations superimposed on exponentially decaying population dynamics. Fast Fourier transformation (FFT) of the observed oscillatory traces revealed a frequency of $590 \mathrm{~cm}^{-1}$. This vibrational mode has been observed previously by both transient absorption and 2DES experiments, ${ }^{26,39}$ and assigned to the ring distortion mode of NB. ${ }^{39}$

\subsection{Application of ultrabroadband 2D electronic spectroscopy to photosynthetic energy transfer: light-harvesting complex II (LHCII)}

In this section, we present an example of how ultrabroadband 2DES can be used to map out energy transfer pathways in photosynthetic light harvesting systems. Light-harvesting complex II (LHCII) in photosystem II is the most abundant pigment-protein complex in higher plants and green algae, responsible for the absorption of over half of the total radiation used for plant photosynthesis on earth. ${ }^{40}$ It serves the principal light-harvesting role of capturing solar energy and funneling it to the reaction center with near-unity quantum efficiency.

In nature, LHCII is found as a trimer with three similar monomeric subunits. ${ }^{41}$ Figure $3 \mathrm{~A}$ shows a structural model of the three-dimensional organization of pigments in an LHCII trimer embedded in a protein matrix. ${ }^{42}$ Each monomeric subunit consists of eight chlorophyll $a(\mathrm{Chl} a)$ and six chlorophyll $b(\mathrm{Chl} b)$ pigments, which are responsible for absorbing sunlight at $600-700 \mathrm{~nm}$ (Q bands) and at wavelengths shorter than $\sim 480 \mathrm{~nm}(\mathrm{~B}$ bands). Carotenoids (two luteins, one neoxanthin, and one violaxanthin in a monomer under low light condition) complement the solar energy absorption by primarily absorbing at $400-550 \mathrm{~nm}$. The absorption spectrum of trimeric LHCII is shown in Figure 3B. While intense B bands of chlorophylls (435 nm for Chl $a$ and $472 \mathrm{~nm}$ for $\mathrm{Chl} b$ ) dominate the blue side of the absorption spectrum, a shoulder peak is observed at $490 \mathrm{~nm}$, which corresponds to the 0-0 vibronic band of the optically allowed $\mathrm{S}_{2}$ transition of carotenoids. ${ }^{43}$ Q $\mathrm{y}$ bands of Chl $b$ and Chl $a$ appear at 652 and $675 \mathrm{~nm}$, respectively. The broad, weak absorption at $500-620$ is assigned to be $\mathrm{Q}_{\mathrm{x}}$ transitions of chlorophylls and higher vibronic states of $\mathrm{Q}_{\mathrm{y}}{ }^{44}$ The $\mathrm{S}_{1}$ transition of carotenoids is optically forbidden, but is suggested to lie close to the $\mathrm{Q}_{\mathrm{y}}$ energy level of $\mathrm{Chl} a{ }^{44}$ Presence of additional dark intermediate states $\left(\mathrm{S}_{\mathrm{x}}\right.$ and $\left.\mathrm{S}^{*}\right)$ between $\mathrm{S}_{2}$ and $\mathrm{S}_{1}$ states has also been postulated, although their nature and function still remain controversial. ${ }^{45}$
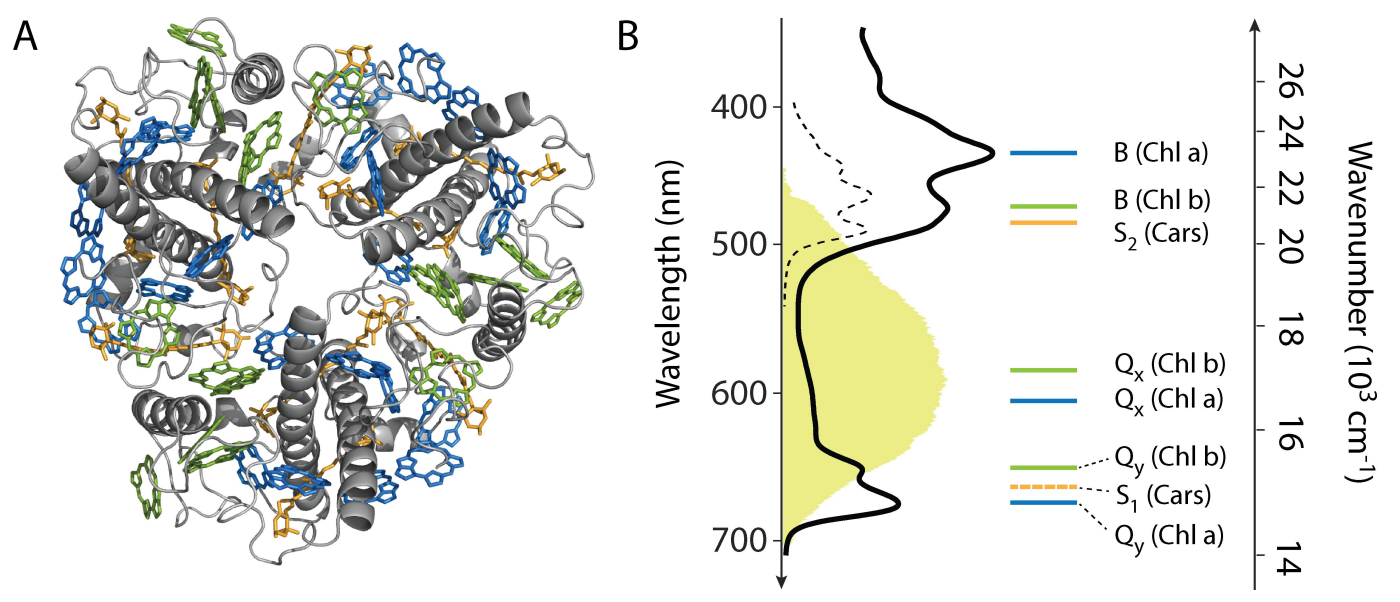

Figure 3. (A) Structural model of LHCII trimer with Chl $a$ shown in blue, Chl $b$ shown in green, and carotenoids shown in orange (PDB code: 1rwt). ${ }^{42}$ (B) Linear absorption spectrum of LHCII (black line) overlaid with the laser spectrum used in 2DES measurements (green area). The dashed spectrum corresponds to the linear absorption of carotenoids (here, violaxanthin is shown as an example; not to scale). The horizontal bars on the right display the energy levels corresponding to electronic transitions of chlorophylls and carotenoids observed in LHCII. 
The pathways and dynamics of energy transfer in LHCII have extensively been investigated by many different ultrafast spectroscopic techniques, such as transient absorption, ${ }^{46-48}$ fluorescence upconversion, ${ }^{49}$ three-pulse photon echo peak shift (3PEPS), ${ }^{50,51}$ and more recently $2 \mathrm{DES},{ }^{27-30}$ revealing energy transfer timescales ranging from 100 fs to several tens of picoseconds. In particular, recent $2 \mathrm{DES}$ studies on $\mathrm{LHCII}^{27}$ combined with theoretical modeling of the excitonic energy levels of chlorophylls ${ }^{52}$ provided additional insights into energy migration between different pools of chlorophylls, which is unable to resolve by transient absorption due to the congested energy landscape. However, the spectral window of all of the 2DES studies on energy transfer in LHCII reported so far is limited to only the two $\mathrm{Q}_{\mathrm{y}}$ bands of Chl $a$ and $\mathrm{Chl} b$, thereby losing access to energy relaxation pathways involving higher-lying states. Our 2DES apparatus overcomes this limitation by utilizing ultrabroadband pulses that encompass a much broader range of transitions, including $\mathrm{Q}_{\mathrm{x}}$ states of chlorophylls and $\mathrm{S}_{2}$ transition of carotenoids.

An exemplary ultrabroadband 2D spectrum of LHCII $(T=183 \mathrm{fs})$ is shown in Figure 4A. The spectrum reveals many distinct peaks over the entire frequency map. The lower half of region I, which corresponds to the $\mathrm{Q}_{\mathrm{y}}$ transitions of chlorophyll pigments, exhibits two prominent well-resolved peaks at $\left(\omega_{\tau}, \omega_{t}\right)=\left(15500 \mathrm{~cm}^{-1}\right.$, $\left.15500 \mathrm{~cm}^{-1}\right)$ and $\left(15200 \mathrm{~cm}^{-1}, 15000 \mathrm{~cm}^{-1}\right)$ (peaks 1 and 2 in Figure 4B). The diagonal peak of Chl $a$ that is expected to appear at $\left(\omega_{\tau}, \omega_{t}\right)=\left(14700 \mathrm{~cm}^{-1}, 14700 \mathrm{~cm}^{-1}\right)^{29}$ is not clearly resolved, most likely because this wavelength is at the red edge of our laser spectrum. The blue side of the spectrum, marked as II in Figure 4A contains peaks originating from photoexcitation of the $\mathrm{S}_{2}$ state of carotenoids. Two peaks, both elongated along the $\omega_{t}$ axis below the diagonal, appear at the excitation frequencies of 20150 and $21100 \mathrm{~cm}^{-1}$, which match the 0-0 and 0-1 vibronic frequencies of the $\mathrm{S}_{2}$ absorption of carotenoids. ${ }^{44}$ Thus, our spectra encompass transitions across the visible, enabling simultaneous excitation of both carotenoid and the chlorophyll transitions. This demonstrates the applicability of our ultrabroadband 2DES setup to studies of systems absorbing over a broad frequency range, while maintaining high time and energy resolution.
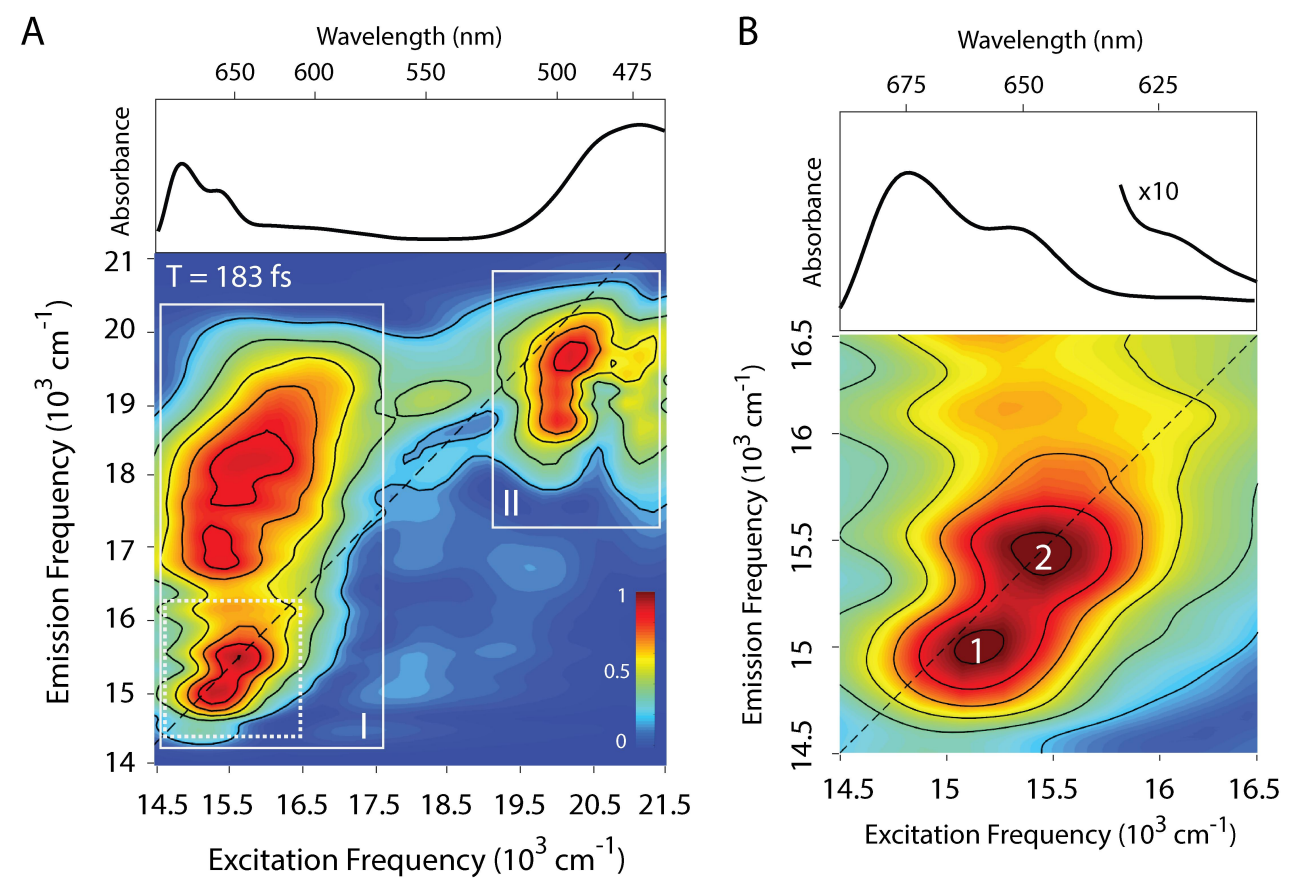

Figure 4. (A) Absolute-value ultrabroadband 2D spectrum of LHCII at $T=183$ fs. The letters I and II mark representative regions where the major peaks are observed for the sake of explanation. The linear absorption of LHCII in the corresponding frequency (wavelength) range is shown on top. (B) Zoom-in of the $2 \mathrm{D}$ spectrum shown in (A) in the $\mathrm{Q}_{\mathrm{y}}$ region (marked as a box with dashed lines in $(\mathrm{A})$ ) with the linear absorption spectrum in the corresponding frequency range. The two representative peaks mentioned in the text are marked with numbers 1 and 2. 


\section{CONCLUSIONS}

To summarize, we described the construction of an improved two-dimensional electronic spectrometer with enhanced spectral coverage that is capable of simultaneously exciting and probing the entire visible region. Expansion of the spectral window renders 2DES an even more powerful tool for visualizing the full energy relaxation pathways of condensed-phase systems, combined with its intrinsically high frequency and time resolution. Applying our ultrabroadband 2DES apparatus to the major light harvesting complex LHCII, we have demonstrated the ability of our setup to map out the pathways of energy flow ranging from higher-lying, carotenoid excited states to the lowest-energy transitions of chlorophylls.

\section{ACKNOWLEDGMENTS}

We acknowledge the Center for Excitonics (an Energy Frontiers Research Center funded by the U.S. Department of Energy, Office of Science, Office of Basic Energy Sciences, through grant no. DE-SC0001088) for support. We would like to thank Dr. Sandra Mosquera Vázquez for assistance in building the ultrabroadband 2DES setup, and Dr. Alberta Pinnola and Prof. Roberto Bassi (University of Verona, Italy) for providing the LHCII samples.

\section{REFERENCES}

[1] Turro, N. J., Ramamurthy, V., and Scaiano, J. C., [Principles of Molecular Photochemistry: An Introduction], University Science Books, Sausalito (2009).

[2] Berera, R., van Grondelle, R., and Kennis, J. T. M., "Ultrafast transient absorption spectroscopy: Principles and application to photosynthetic systems," Photosynth. Res. 101(2), 105-118 (2009).

[3] Xu, J. and Knutson, J. R., "Ultrafast fluorescence spectroscopy via upconversion: Applications to biophysics," Methods Enzymol. 450, 159-183 (2008).

[4] Cho, M., "Coherent two-dimensional optical spectroscopy," Chem. Rev. 108(4), 1331-1418 (2008).

[5] Schlau-Cohen, G. S., Ishizaki, A., and Fleming, G. R., "Two-dimensional electronic spectroscopy and photosynthesis: Fundamentals and applications to photosynthetic light-harvesting," Chem. Phys. 386(1), 1-22 (2011).

[6] Anna, J. M., Song, Y., Dinshaw, R., and Scholes, G. D., "Two-dimensional electronic spectroscopy for mapping molecular photophysics," Pure Appl. Chem. 85(7), 1307-1319 (2013).

[7] Jonas, D. M., "Two-dimensional femtosecond spectroscopy," Annu. Rev. Phys. Chem. 54(1), 425-463 (2003).

[8] Brixner, T., Stenger, J., Vaswani, H. M., Cho, M., Blankenship, R. E., and Fleming, G. R., "Twodimensional spectroscopy of electronic couplings in photosynthesis," Nature 434(7033), 625-628 (2005).

[9] Cheng, Y.-C. and Fleming, G. R., "Dynamics of light harvesting in photosynthesis," Annu. Rev. Phys. Chem. 60, 241-262 (2009).

[10] Lewis, K. L. M. and Ogilvie, J. P., "Probing photosynthetic energy and charge transfer with two-dimensional electronic spectroscopy," J. Phys. Chem. Lett. 3(4), 503-510 (2012).

[11] Stiopkin, I., Brixner, T., Yang, M., and Fleming, G. R., "Heterogeneous exciton dynamics revealed by two-dimensional optical spectroscopy," J. Phys. Chem. B 110(40), 20032-20037 (2006).

[12] Arias, D. H., Stone, K. W., Vlaming, S. M., Walker, B. J., Bawendi, M. G., Silbey, R. J., Bulovic, V., and Nelson, K. A., "Thermally-limited exciton delocalization in superradiant molecular aggregates," J. Phys. Chem. B 117(16), 4553-4559 (2012).

[13] Lim, J., Paleček, D., Caycedo-Soler, F., Lincoln, C. N., Prior, J., von Berlepsch, H., Huelga, S. F., Plenio, M. B., Zigmantas, D., and Hauer, J., "Vibronic origin of long-lived coherence in an artificial molecular light harvester," Nat. Commun. 6, 7755 (2015).

[14] Caram, J. R., Fidler, A. F., and Engel, G. S., "Excited and ground state vibrational dynamics revealed by two-dimensional electronic spectroscopy," J. Chem. Phys. 137(2), 024507 (2012).

[15] Cassette, E., Pensack, R. D., Mahler, B., and Scholes, G. D., "Room-temperature exciton coherence and dephasing in two-dimensional nanostructures," Nat. Commun. 6, 6086 (2015).

[16] Mehlenbacher, R. D., McDonough, T. J., Grechko, M., Wu, M.-Y., Arnold, M. S., and Zanni, M. T., "Energy transfer pathways in semiconducting carbon nanotubes revealed using two-dimensional white-light spectroscopy," Nat. Commun. 6, 6732 (2015). 
[17] Bakulin, A. A., Morgan, S. E., Kehoe, T. B., Wilson, M. W. B., Chin, A. W., Zigmantas, D., Egorova, D., and Rao, A., "Real-time observation of multiexcitonic states in ultrafast singlet fission using coherent 2D electronic spectroscopy," Nat. Chem. 8(1), 16-23 (2016).

[18] De Sio, A., Troiani, F., Maiuri, M., Réhault, J., Sommer, E., Lim, J., Huelga, S. F., Plenio, M. B., Rozzi, C. A., Cerullo, G., et al., "Tracking the coherent generation of polaron pairs in conjugated polymers," Nat. Commun. 7, 13742 (2016).

[19] Wilhelm, T., Piel, J., and Riedle, E., "Sub-20-fs pulses tunable across the visible from a blue-pumped single-pass noncollinear parametric converter," Opt. Lett. 22(19), 1494-1496 (1997).

[20] Fuller, F. D. and Ogilvie, J. P., "Experimental implementations of two-dimensional Fourier transform electronic spectroscopy," Annu. Rev. Phys. Chem. 66, 667-690 (2015).

[21] Hauri, C. P., Kornelis, W., Helbing, F. W., Heinrich, A., Couairon, A., Mysyrowicz, A., Biegert, J., and Keller, U., "Generation of intense, carrier-envelope phase-locked few-cycle laser pulses through filamentation," Appl. Phys. B 79(6), 673-677 (2004).

[22] Hagemann, F., Gause, O., Wöste, L., and Siebert, T., "Supercontinuum pulse shaping in the few-cycle regime," Opt. Express 21(5), 5536-5549 (2013).

[23] Zheng, H., Caram, J. R., Dahlberg, P. D., Rolczynski, B. S., Viswanathan, S., Dolzhnikov, D. S., Khadivi, A., Talapin, D. V., and Engel, G. S., "Dispersion-free continuum two-dimensional electronic spectrometer," Appl. Opt. 53(9), 1909-1917 (2014).

[24] Spokoyny, B., Koh, C. J., and Harel, E., "Stable and high-power few cycle supercontinuum for 2D ultrabroadband electronic spectroscopy," Opt. Lett. 40(6), 1014-1017 (2015).

[25] Ma, X., Dostál, J., and Brixner, T., "Broadband 7-fs diffractive-optic-based 2D electronic spectroscopy using hollow-core fiber compression," Opt. Express 24(18), 20781-20791 (2016).

[26] Seiler, H., Palato, S., Schmidt, B. E., and Kambhampati, P., "Simple fiber-based solution for coherent multidimensional spectroscopy in the visible regime," Opt. Lett. 42(3), 643-646 (2017).

[27] Schlau-Cohen, G. S., Calhoun, T. R., Ginsberg, N. S., Read, E. L., Ballottari, M., Bassi, R., van Grondelle, R., and Fleming, G. R., "Pathways of energy flow in LHCII from two-dimensional electronic spectroscopy," J. Phys. Chem. B 113(46), 15352-15363 (2009).

[28] Wells, K. L., Lambrev, P. H., Zhang, Z., Garab, G., and Tan, H.-S., "Pathways of energy transfer in LHCII revealed by room-temperature 2D electronic spectroscopy," Phys. Chem. Chem. Phys. 16(23), 11640-11646 (2014).

[29] Duan, H.-G., Stevens, A. L., Nalbach, P., Thorwart, M., Prokhorenko, V. I., and Miller, R. J. D., "Twodimensional electronic spectroscopy of light-harvesting complex II at ambient temperature: A joint experimental and theoretical study," J. Phys. Chem. B 119(36), 12017-12027 (2015).

[30] Enriquez, M. M., Akhtar, P., Zhang, C., Garab, G., Lambrev, P. H., and Tan, H.-S., "Energy transfer dynamics in trimers and aggregates of light-harvesting complex II probed by 2D electronic spectroscopy," J. Chem. Phys. 142(21), 212432 (2015).

[31] Pervak, V., Ahmad, I., Trubetskov, M. K., Tikhonravov, A. V., and Krausz, F., "Double-angle multilayer mirrors with smooth dispersion characteristics," Opt. Express 17(10), 7943-7951 (2009).

[32] Trebino, R., DeLong, K. W., Fittinghoff, D. N., Sweetser, J. N., Krumbügel, M. A., Richman, B. A., and Kane, D. J., "Measuring ultrashort laser pulses in the time-frequency domain using frequency-resolved optical gating," Rev. Sci. Instrum. 68(9), 3277-3295 (1997).

[33] Brixner, T., Mančal, T., Stiopkin, I. V., and Fleming, G. R., "Phase-stabilized two-dimensional electronic spectroscopy," J. Chem. Phys. 121(9), 4221-4236 (2004).

[34] Hamm, P. and Zanni, M. T., [Concepts and Methods of 2D Infrared Spectroscopy], Cambridge University Press, New York (2011).

[35] Zhang, Y., Meyer, K., Ott, C., and Pfeifer, T., "Passively phase-stable, monolithic, all-reflective twodimensional electronic spectroscopy based on a four-quadrant mirror," Opt. Lett. 38(3), 356-358 (2013).

[36] Heisler, I. A., Moca, R., Camargo, F. V., and Meech, S. R., "Two-dimensional electronic spectroscopy based on conventional optics and fast dual chopper data acquisition," Rev. Sci. Instrum. 85(6), 063103 (2014).

[37] Caffarri, S., Croce, R., Breton, J., and Bassi, R., "The major antenna complex of photosystem II has a xanthophyll binding site not involved in light harvesting," J. Biol. Chem. 276(38), 35924-35933 (2001). 
[38] Turner, D. B., Wilk, K. E., Curmi, P. M., and Scholes, G. D., "Comparison of electronic and vibrational coherence measured by two-dimensional electronic spectroscopy," J. Phys. Chem. Lett. 2(15), 1904-1911 (2011).

[39] Fragnito, H. L., Bigot, J.-Y., Becker, P. C., and Shank, C. V., "Evolution of the vibronic absorption spectrum in a molecule following impulsive excitation with a 6 fs optical pulse," Chem. Phys. Lett. 160(2), 101-104 (1989).

[40] Barros, T. and Kühlbrandt, W., "Crystallisation, structure and function of plant light-harvesting complex II,” BBA-Bioenergetics 1787(6), 753-772 (2009).

[41] Lambrev, P. H., Várkonyi, Z., Krumova, S., Kovács, L., Miloslavina, Y., Holzwarth, A. R., and Garab, G., "Importance of trimer-trimer interactions for the native state of the plant light-harvesting complex II," BBA-Bioenergetics 1767(6), 847-853 (2007).

[42] Liu, Z., Yan, H., Wang, K., Kuang, T., Zhang, J., Gui, L., An, X., and Chang, W., "Crystal structure of spinach major light-harvesting complex at $2.72 \AA$ resolution," Nature 428(6980), 287-292 (2004).

[43] Ruban, A. V., Pascal, A. A., Robert, B., and Horton, P., "Configuration and dynamics of xanthophylls in light-harvesting antennae of higher plants - spectroscopic analysis of isolated light-harvesting complex of photosystem II and thylakoid membranes," J. Biol. Chem. 276(27), 24862-24870 (2001).

[44] Polívka, T., Zigmantas, D., Sundström, V., Formaggio, E., Cinque, G., and Bassi, R., "Carotenoid $\mathrm{S}_{1}$ state in a recombinant light-harvesting complex of photosystem II," Biochemistry 41(2), 439-450 (2002).

[45] Polívka, T. and Sundström, V., "Dark excited states of carotenoids: Consensus and controversy," Chem. Phys. Lett. 477(1), 1-11 (2009).

[46] Connelly, J. P., Müller, M. G., Hucke, M., Gatzen, G., Mullineaux, C. W., Ruban, A. V., Horton, P., and Holzwarth, A. R., "Ultrafast spectroscopy of trimeric light-harvesting complex II from higher plants," $J$. Phys. Chem. B 101(10), 1902-1909 (1997).

[47] Gradinaru, C. C., Özdemir, S., Gülen, D., van Stokkum, I. H., van Grondelle, R., and van Amerongen, H., "The flow of excitation energy in LHCII monomers: implications for the structural model of the major plant antenna," Biophys. J. 75(6), 3064-3077 (1998).

[48] Croce, R., Müller, M. G., Bassi, R., and Holzwarth, A. R., "Carotenoid-to-chlorophyll energy transfer in recombinant major light-harvesting complex (LHCII) of higher plants. I. Femtosecond transient absorption measurements," Biophys. J. 80(2), 901-915 (2001).

[49] Du, M., Xie, X., Mets, L., and Fleming, G. R., "Direct observation of ultrafast energy-transfer processes in light harvesting complex II," J. Phys. Chem. 98(17), 4736-4741 (1994).

[50] Agarwal, R., Krueger, B. P., Scholes, G. D., Yang, M., Yom, J., Mets, L., and Fleming, G. R., "Ultrafast energy transfer in LHC-II revealed by three-pulse photon echo peak shift measurements," J. Phys. Chem. B 104(13), 2908-2918 (2000).

[51] Salverda, J. M., Vengris, M., Krueger, B. P., Scholes, G. D., Czarnoleski, A. R., Novoderezhkin, V., van Amerongen, H., and van Grondelle, R., "Energy transfer in light-harvesting complexes LHCII and CP29 of spinach studied with three pulse echo peak shift and transient grating," Biophys. J. 84(1), 450-465 (2003).

[52] Novoderezhkin, V., Marin, A., and van Grondelle, R., "Intra-and inter-monomeric transfers in the light harvesting LHCII complex: the Redfield-Förster picture," Phys. Chem. Chem. Phys. 13(38), 17093-17103 (2011). 